\title{
Necrotizing Pseudomonas chondritis after piercing of the upper ear
}

\author{
Todd C. Lee MD, Wayne L. Gold MD
}

1 previously healthy 15 -year-old girl had the upper portion of her left ear pierced at a local boutique. The ear was cleansed with an individually wrapped $70 \%$ alcohol swab and pierced with an individually wrapped sterile straight needle. A single metallic stud was removed from a plastic packet and was placed into the hole. The piercer wore gloves after washing both hands. Following the procedure, the patient cleansed the site using soap in combination with a skin cleanser (Spectro Jel, GlaxoSmithKline, Oakville, Ontario) and using boiled water on most occasions. She took showers without covering her ear. The ear was not exposed to fresh water or water in swimming pools.

Within 24 hours after the piercing, the site became erythematous, painful and swollen. The patient tried to treat the ear with a solution of boiled water and salt. Over the following four days, she experienced progressive pain and swelling followed by spontaneous purulent discharge from the site, prompting her to seek medical attention. She reported no fever. The stud was removed, the posterior upper helix was incised, and pus was drained. Empirical treatment with cephalexin was started for the management of an abscess with surrounding cellulitis. After several days without improvement, a second drainage procedure was done, and intravenous treatment with cefazolin and clindamycin was started. A specimen obtained for culture yielded a pure growth of Pseudomonas aeruginosa that was sensitive to ciprofloxacin, ceftazidime and piperacillin-tazobactam. Antibiotics were changed to intravenous ciprofloxacin. After three days, she was discharged home with a prescription for oral ciprofloxacin.

Despite two weeks of oral ciprofloxacin treatment, the patient continued to experience pain, swelling and discharge from her ear. A consultation with an infectious diseases specialist was requested. On examination, the left ear was tender, swollen and erythematous with a mass $(2 \times$ $2 \times 1 \mathrm{~cm}$ ) overlying the posterior aspect of the upper helix (Figure 1). There was seropurulent drainage through the earlier incision. Urgent consultation with a plastic surgeon was requested. Under local anesthetic, a small amount of pus was drained through a posterior incision, and a hematoma was removed through a separate anterior incision. The wound was allowed to heal by secondary intention with daily packing. Because the infection had progressed despite the ciprofloxacin treatment, the antimicrobial regimen was changed from ciprofloxacin to piperacillin-tazobactam (4.5 g intravenously every eight hours for three weeks). However, the failure to respond was thought to be more likely the result of inadequate initial drainage than a failure of antimicrobial therapy.

At the completion of treatment, minimal deformity of the helix remained (Figure 2). A decision regarding reconstructive surgery to improve the cosmetic appearance of the ear has been deferred to allow for further healing.

\section{Discussion}

Body piercing, including piercing of the auricular cartilage in the upper ear, has become increasingly popular. ${ }^{1,2}$ The practice of body piercing is largely unregulated, and the training of piercers about infection control varies. In addition, there is considerable variation in instructions for postprocedural care of the piercing site..$^{1-3}$ Because of the trauma of the procedure, body piercing at any site

\section{KEY POINTS}

- Organisms that cause infection at piercing sites may be introduced at the time of the procedure or during postprocedural care.

- Infections are generally caused by commensal flora at the site of the piercing.

- Piercings of auricular cartilage carry a greater risk of infection than soft-tissue piercings do.

- Infection at the site of an upper-ear piercing may be caused by Pseudomonas aeruginosa, which should be covered in the initial, empirical antimicrobial regimen. 
may result in local inflammation and swelling. Metal hypersensitivity reactions may also occur. ${ }^{3}$ Although infections related to body piercing are infrequently reported in the literature, one estimate has placed the incidence at $11 \%-24 \%$. Organisms that cause infection may be introduced at the time of piercing as a result of improper cleansing of the site or through the use of nonsterile equipment. In these cases, infection is generally caused by local skin flora. Infections may also be introduced during postprocedural care of the site. Pseudomonal infection of the ear after piercing has occurred with the use of contaminated cleansing solution. ${ }^{4}$ General principles to prevent infection after piercing, based on expert opinion, are included in Box $1 .^{1-3}$

\section{Microbiology}

Organisms responsible for infection of the external ear differ by location of the piercing (Box 2).

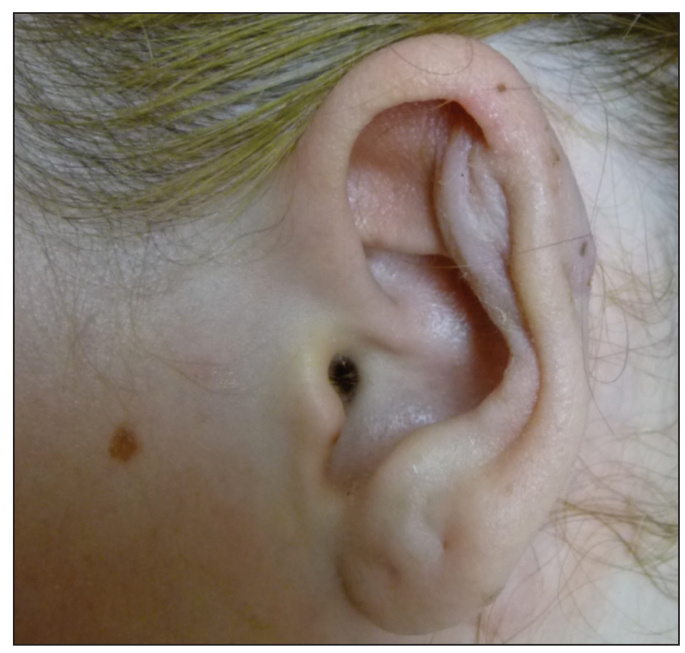

Figure 1: The left ear of a 15-year-old girl, showing auricular chondritis and abscess after piercing.

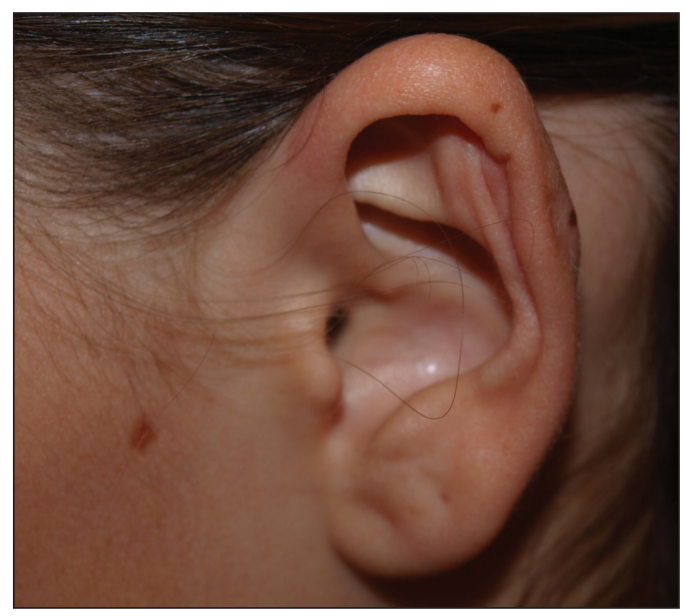

Figure 2: Residual deformity of the helix three months after surgical débridement and treatment with broad-spectrum antibiotics.
Because micro-organisms may be introduced at the time of the piercing, an understanding of the commensal organisms at piercing sites can help when choosing empirical antimicrobial treatment. Staphylococcus aureus is the organism most frequently reported with infected piercings of the skin, because it is part of the normal skin flora. For piercings involving the mouth, oral flora such as $\alpha$-hemolytic streptococci and anaerobes are most frequently encountered. ${ }^{5}$

Although infection can complicate piercing at any site, piercings of cartilaginous tissue carry an increased risk of infection over soft-tissue piercings because of the relatively avascular nature of cartilage.${ }^{3,6}$ Early recognition of this potentially serious complication of auricular piercing is important to reduce the extent of cartilage damage. Necrotizing pseudomonal infection of the auricular cartilage is a rare complication of upperear piercing. Pseudomonas chondritis usually presents as cellulitis of the helix within one week after piercing. ${ }^{5,7,8}$ Later presentations have also been described. ${ }^{7}$ Abscess formation and pressureinduced injury to the cartilage may follow. Necrosis of the cartilage is associated with undesirable cosmetic outcomes; the development of a "cauliflower ear" is the most serious complication. ${ }^{2,3}$

\section{Epidemiology}

The incidence of infection after piercing of the upper ear has not been accurately calculated. A

\section{Box 1: Recommendations for the} prevention of infection after piercing*

- The piercer should wear sterile gloves after washing his or her hands with soap and water.

- A single-use, nonreusable setup should be used to perform the piercing, and any items that are not disposable should be autoclaved between clients.

- Metal jewellery to be placed at the time of piercing should be autoclaved.

- Before piercing, the skin at the piercing site should be cleansed with an alcohol-based disinfectant intended for single use.

- After piercing, the site should be cleansed daily with an antiseptic solution; otherwise, the piercing site should not be handled until it has healed.

- Exposure of the piercing site to fresh water or water in swimming pools and hot tubs should be avoided until the site has healed.

- The client should be instructed to seek medical attention at the earliest signs of infection, including erythema, pain or discharge from the site.

*These recommendations are based on expert clinical opinion. ${ }^{1-3}$ There is no evidence to support the use of prophylactic antimicrobial treatment to prevent infection at piercing sites. 
survey of otolaryngologists in the State of Oregon in the United States was conducted during the investigation of an outbreak of $P$. aeruginosa infections after commercial upper-ear piercing. The survey showed that, in 1406 years of combined practice, 190 patients with piercing-related auricular chondritis had been seen, of whom 27 (14\%) had infection due to $P$. aeruginosa. ${ }^{8}$ Another study suggested that the overall incidence of auricular chondritis in the United Kingdom increased during the 1990s, coincident with the increasing popularity of this form of body art. ${ }^{2}$

Pseudomonal chondritis after upper-ear piercing may occur sporadically or in outbreaks. ${ }^{2,6,8}$ Previously identified risk factors for infection include the use of disinfectants from multiuse refillable bottles and the use of benzalkonium chloride as opposed to alcohol-based disinfectants to cleanse the skin, because $P$. aeruginosa is resistant to this agent. The use of an open piercing gun as opposed to a sterile straight needle may predispose to an increased risk of infection secondary to shearing of the perichondrium. Furthermore, exposure of the wound to fresh water or water in swimming pools and hot tubs after the procedure has been shown to increase the risk of infection. ${ }^{6-8}$

\section{Management}

No official guidelines exist to assist in the management of infections related to body piercing. However, information from case series and recommendations from experts suggest that the following general principles should be followed. Patients who experience an infection at the site of an upper-ear piercing should remove the jewellery and seek immediate medical attention. If a drainable collection of pus or a hematoma is present, it should be drained, preferably by an experienced plastic surgeon or otolaryngologist, and specimens for culture taken. ${ }^{2}$ Repeated evaluations are required to evacuate accumulating hematomas or abscesses to minimize pressureinduced injury to the cartilage.

Empirical antimicrobial treatment should provide coverage against $P$. aeruginosa as well as

\section{Box 2: Bacteria commonly responsible for infections of the external ear, by site of piercing}

- Lobule: Staphylococcus aureus*

- Helix: S. aureus, * Pseudomonas aeruginosa†

- Tragus: $S$. aureus, ${ }^{*} P$. aeruginosat

*Including methicillin-resistant $S$. aureus in the appropriate epidemiologic settings. ${ }^{9}$

tEmpirical antimicrobial treatment with coverage against $P$. aeruginosa should be provided for piercing-related infections involving cartilaginous tissue. normal skin flora, including $S$. aureus. In certain epidemiologic settings, treatment may also include coverage against community-associated strains of methicillin-resistant S. aureus. Populations at increased risk of community-associated methicillin-resistant $S$. aureus infection include athletes (of mainly contact sports), injection drug users, men who have sex with men, inmates of correctional institutions, and native or Aboriginal populations. ${ }^{9}$ A reasonable empirical antimicrobial regimen might include antipseudomonal coverage with ciprofloxacin in combination with cefazolin for methicillin-sensitive $S$. aureus or vancomycin for methicillin-resistant $S$. aureus. Monotherapy with piperacillin-tazobactam will provide coverage against both $P$. aeruginosa and methicillin-sensitive $S$. aureus. Antimicrobial treatment should be continued for two to four weeks. ${ }^{4,10}$ Ciprofloxacin (750 mg orally twice daily) is appropriate for monotherapy if $P$. aeruginosa is isolated and the isolate is susceptible.

Despite appropriate antimicrobial treatment and the drainage of pus or hematoma, persistent deformities of the helix may develop, as seen in our patient. Cosmetic outcomes may be improved by reconstruction of the ear with autogenous tissue. ${ }^{3}$ Decisions regarding corrective surgery should be made on an individual basis.

\section{References}

1. Tweeten SS, Rickman L. Infectious complications of body piercing. Clin Infect Dis 1998;26:735-40.

2. Hanif J, Frosh A, Marnane C, et al. High ear piercing and the rising risk of perichondritis of the pinna. BMJ 2001;322:906-7.

3. Cicchetti S, Skillman J, Gault DT. Piercing of the upper ear: a simple infection, a difficult reconstruction. Br J Plast Surg 2002; 55:194-7.

4. Pier GB, Ramphal R. Pseudomonas aeruginosa. In: Mandell GL, Bennett JE, Dolin R, editors. Principles and practice of infectious diseases. 6th ed. Philadelphia (PA): Elsevier Churchill Livingstone; 2005. p. 2587-615.

5. Yu CHY, Minnema B, Gold WL. Bacterial infections complicating tongue piercing. Can J Infect Dis Med Microbiol 2010; 21:e70-4.

6. Fisher CG, Kacica MA, Bennett NM. Risk factors for cartilage infections of the ear. Am J Prev Med 2003;29:204-9.

7. Sandhu A, Gross M, Wylie J, et al. Pseudomonas aeruginosa necrotizing chondritis complicating high helical ear piercing case report: clinical and public health perspectives. Can J Public Health 2007;98:74-7.

8. Keene WE, Markum AC, Samadpour M. Outbreak of Pseudomonas aeruginosa infections caused by commercial piercing of upper ear cartilage. JAMA 2004;291:981-5.

9. Hawkes M, Barton M, Conly J, et al. Community-associated MRSA: superbug at our doorstep. CMAJ 2007;176:54-6.

10. Rowshan HH, Keith K, Baur D, et al. Pseudomonas aeruginosa infection of the auricular cartilage caused by "high ear piercing": a case report and review of the literature. J Oral Maxillofac Surg 2008;66:543-6.

Affiliations: From the Division of Infectious Diseases, University Health Network, University of Toronto, Toronto, Ont.

Contributors: All of the authors contributed substantially to the drafting of the article and approved the final version submitted for publication.

Acknowledgement: The authors thank Dr. Toni Zhong for her assistance in the management of this patient and for providing the photograph in Figure 1. 